Pierre-Alain Duc, Jonathan Braine and Elias Brinks, eds.

\title{
Molecular Gas and Star Formation in the NGC 3077 Tidal Arm
}

\author{
Fabian Walter \\ NRAO, P.O. Box O, Socorro, NM 87801, USA
}

Crystal Martin

UC Santa Barbara, Santa Barbara, CA 93106-9530, USA

Jürgen Ott

CSIRO, ATNF, P.O. Box 76, Epping NSW 1710, Australia

Andreas Heithausen

University of Bonn, Auf dem Hügel 71, 53121 Bonn, Germany

\begin{abstract}
.
We report the discovery of extended star formation in the prominent tidal arms near NGC 3077 (member of the M 81 triplet). 36 faint compact star forming regions were identified, covering an area of $4 \times 6 \mathrm{kpc}^{2}$. HII regions are only found near the southern rim of the tidal $\mathrm{HI}$ arm where the HI column density reaches values above $1 \times 10^{21} \mathrm{~cm}^{-2}$. This threshold is very similar to what is found in 'normal' galactic environments. We derive a total star formation rate of $2.6 \times 10^{-3} M_{\odot} \mathrm{yr}^{-1}$ in the tidal feature. We also present the first high-resolution observations of molecular gas in this region. The molecular gas emission can be separated into at least 5 distinct complexes most of which do not coincide with sites of star formation. The reservoir of neutral and molecular gas in the tidal arm is huge $\left(\sim 5 \times 10^{8} M_{\odot}\right)$; star formation may continue at the given rate for a Hubble time. We conclude that wide-spread low-level star formation may be a common phenomenon in tidal HI tails, however it will be difficult to detect in interacting systems that are further away.
\end{abstract}

\section{Introduction}

It has been suggested that at least a fraction of today's dwarf galaxies was created in the tidal debris of interacting galaxies at large redshift (so-called 'tidal dwarf galaxies', Hunsberger et al. 1996; Hunter et al. 2000; Okazaki \& Taniguchi 2000). Since this process took place in the distant past it is difficult to study the mechanisms that presumably lead to the formation of these tidal dwarfs. More nearby, interacting systems give us the unique opportunity to study the properties of tidal features which may lead to the formation of tidal dwarfs in 


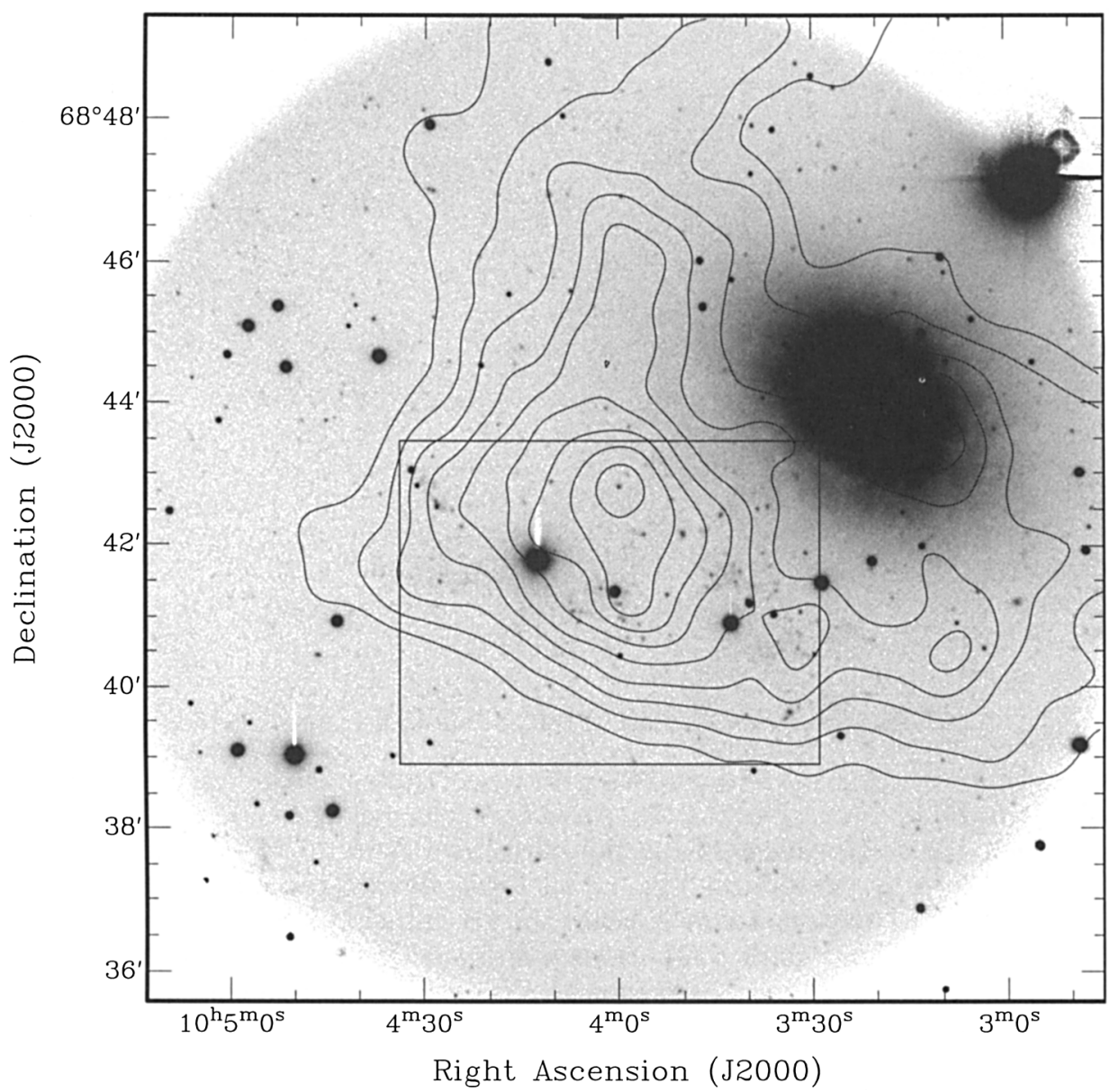

Figure 1. Broad band image of NGC 3077 and its surrounding. Overlaid are the $\mathrm{HI}$ contours (smoothed to $40^{\prime \prime}$ ) which show the orientation of the tidal arms. The box marks the region where we detected HII regions in the tidal arm (see Fig. 2).

detail. E.g., it is of interest which conditions (e.g., atomic and molecular gas content) need to be present before star formation can start in tidal arms.

In a number of cases tidal dwarf galaxies have been detected in tidal tails of interacting galaxies (e.g., Mirabel et al. 1992, Duc et al. 2000). However, most of the tidal dwarfs studied so far are typically bright and have magnitudes ranging from -14 to $-19\left(\mathrm{M}_{B}\right.$, Mirabel et al. 1992) implying that these objects are already fairly evolved. This is obviously a selection effect since only those tidal dwarf galaxies at large distances can be detected which are bright enough.

The nearby M 81 triplet (consisting of M 82, M 81 and NGC 3077, see Yun et al. 1993 for an HI map) is an ideal testbed to study tidal arms around interacting galaxies in detail. The impressive tidal arm near NGC 3077 is especially 


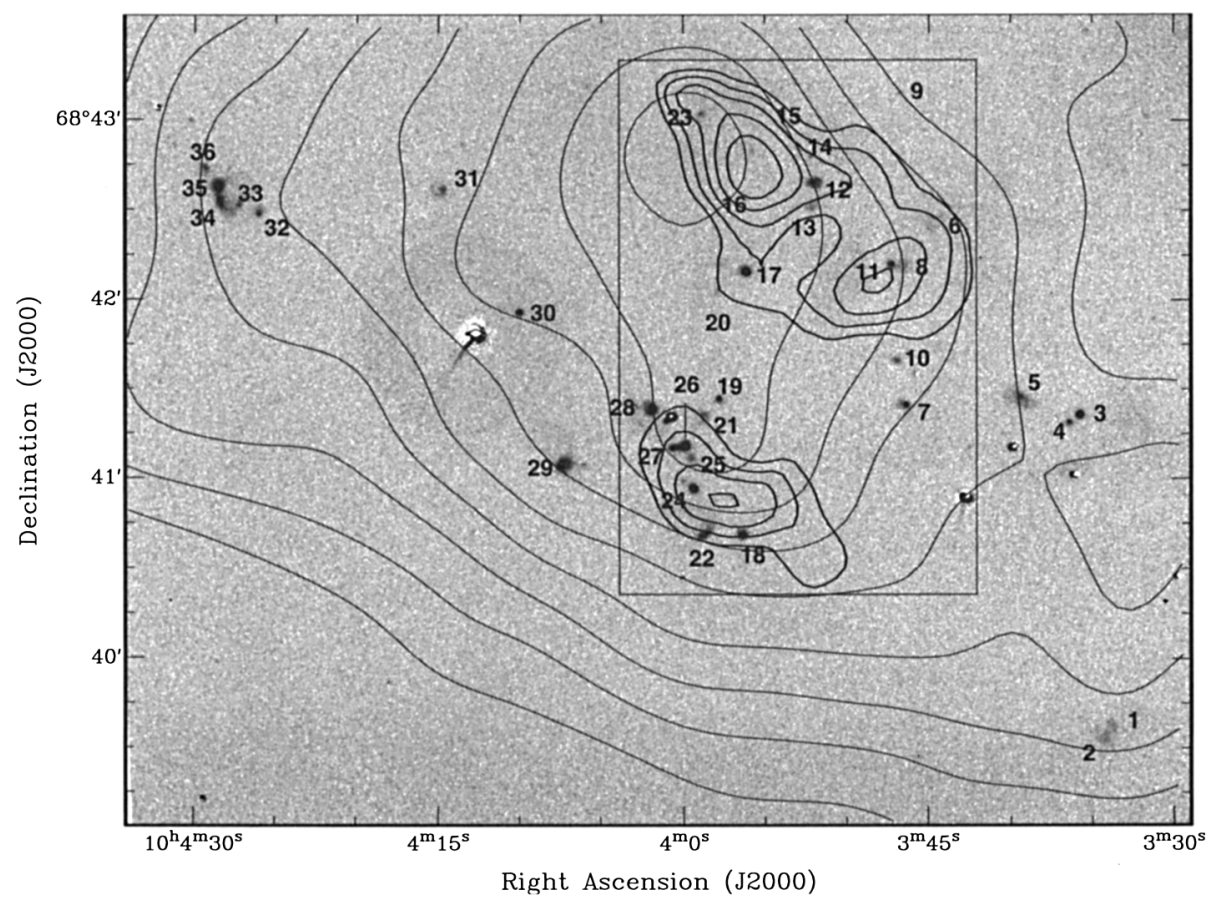

Figure 2. $\mathrm{H} \alpha$ image of the tidal arm near NGC 3077 (the region is indicated by the box in Fig. 1. In total, 37 HII regions were cataloged. The thin contours indicate the HI emission (smoothed to $40^{\prime \prime}$ resolution). The thick contours represent the $\mathrm{CO}(1-0)$ emission at $22^{\prime \prime}$ $(350 \mathrm{pc})$ resolution as observed with the IRAM $30 \mathrm{~m}$ telescope (for details see Heithausen \& Walter 2000).

interesting (total HI mass: $5 \times 10^{8} M_{\odot}$, Walter et al. 2001). According to various numerical simulations (Thomasson \& Donner 1993, Brouillet et al. 1991, Yun et al. 1993) this tail has been created by a recent $\left(\sim 3 \times 10^{8} \mathrm{yr}\right)$ interaction with M 81. Barbieri et al. (1974) detected 'a fragmentary complex of almost stellar objects' in this region (the 'Garland', see also Karachentsev et al. 1985, Sharina 1991, Sakai \& Madore 2001). The presence of extended molecular gas in this tidal feature has been reported by Walter \& Heithausen (1999) and Heithausen \& Walter (2000). Here we report the discovery of wide-spread low-level star formation in this tidal tail near NGC 3077.1

\section{Observations}

We have obtained deep wide-field imaging of the region including NGC 3077 and the prominent tidal arm with both the $2.2 \mathrm{~m}$ telescope at Calar Alto as well as with the $2 \mathrm{~m}$ telescope at Kitt Peak. The new high-resolution observations of the molecular gas phase presented here were obtained with the OVRO millimeter interferometer located near Bishop, CA. 


\section{Discovery of HII Regions in the Tidal Arm}

Fig. 1 shows a B-band image of the region around NGC 3077. The contours show the distribution of neutral hydrogen (HI, smoothed to $40^{\prime \prime}$ resolution, Walter et al. 2001). Fig. 2 is a blown-up $\mathrm{H} \alpha$-map of Fig. 1 and shows the HII regions in the tidal arm near NGC 3077. In total, we cataloged 36 HII regions - all are associated with the prominent tidal HI arm near NGC 3077. The total $\mathrm{H} \alpha$ flux (luminosity) for all the HII regions is: $\mathrm{F}(\mathrm{H} \alpha)=1.88 \times 10^{-13} \mathrm{erg} \mathrm{s}^{-1} \mathrm{~cm}^{-2}$ $\left(\mathrm{L}(\mathrm{H} \alpha)=2.91 \times 10^{38} \mathrm{erg} \mathrm{s}^{-1}\right)$. This corresponds to a total star formation rate (SFR) in the entire tidal arm of SFR $=2.6 \times 10^{-3} \mathrm{M}_{\odot} \mathrm{yr}^{-1}$ (we used SFR(total) $=\mathrm{L}(\mathrm{H} \alpha) / 1.12 \times 10^{41} \mathrm{erg} \mathrm{s}^{-1}\left[\mathrm{M}_{\odot} \mathrm{yr}^{-1}\right]$, Kennicutt, 1983)

\subsection{Comparison to Atomic and Molecular Gas}

The HII regions are distributed over a huge area of $4 \times 6 \mathrm{kpc}^{2}=24 \mathrm{kpc}^{2}$. HII regions are only found where the HI column density reaches values $>1.0 \times$ $10^{21} \mathrm{~cm}^{-2}$. The star forming regions are however not randomly distributed across the entire tidal feature: most of the HII regions in the tidal arm seem to follow the southern rim of the tidal arm. Only one HII region is found in the region where the HI shows multiple velocity components (between regions 30 and 32, Walter et al. 2001).

The thick lines in Fig. 1 present the distribution of molecular gas in the tidal features as traced by the $\mathrm{CO}(1 \rightarrow 0)$ emission observed with the IRAM $30 \mathrm{~m}$ telescope $\left(22^{\prime \prime}, \sim 350 \mathrm{pc}\right.$ resolution; Heithausen \& Walter 2000). Note that the southern, more compact region, where molecular gas was detected coincides with the highest density of star formation.

\subsection{New high-resolution CO observations}

Figure 3 shows our new high-resolution OVRO observations overlaid on a subregion of the $\mathrm{H} \alpha$ image of Fig. 2. Clearly, the $\mathrm{CO}$ emission breaks up into smaller individual clumps. Note that the OVRO interferometer observations (resolution: $5^{\prime \prime}, \sim 75 \mathrm{pc}$ ) are only sensitive to compact structures and do not recover diffuse extended emission present in the IRAM $30 \mathrm{~m}$ data shown in Fig. 2. In the higher resolution $\mathrm{CO}$ data no clear correlation between the location of the molecular gas and the position of the HII regions is evident. Only in one case is a star forming region directly associated with a molecular cloud. We are currently combining our results of the high-resolution OVRO study with the single dish $30 \mathrm{~m}$ observations.

\section{Discussion}

We present the discovery of wide-spread star formation and new high resolution observations of the molecular gas phase in the tidal feature near NGC 3077. The total star formation rate in the tidal arm is $\mathrm{SFR}=2.6 \times 10^{-3} \mathrm{M}_{\odot} \mathrm{yr}^{-1}$. These properties make the complexes by far the weakest star forming regions detected in tidal arms around galaxies so far.

No clear correlation exists between the sites of on-going star formation and gas surface densities (both molecular and atomic): HII regions are in general not only found where the HI or molecular surface density is highest. However, star 


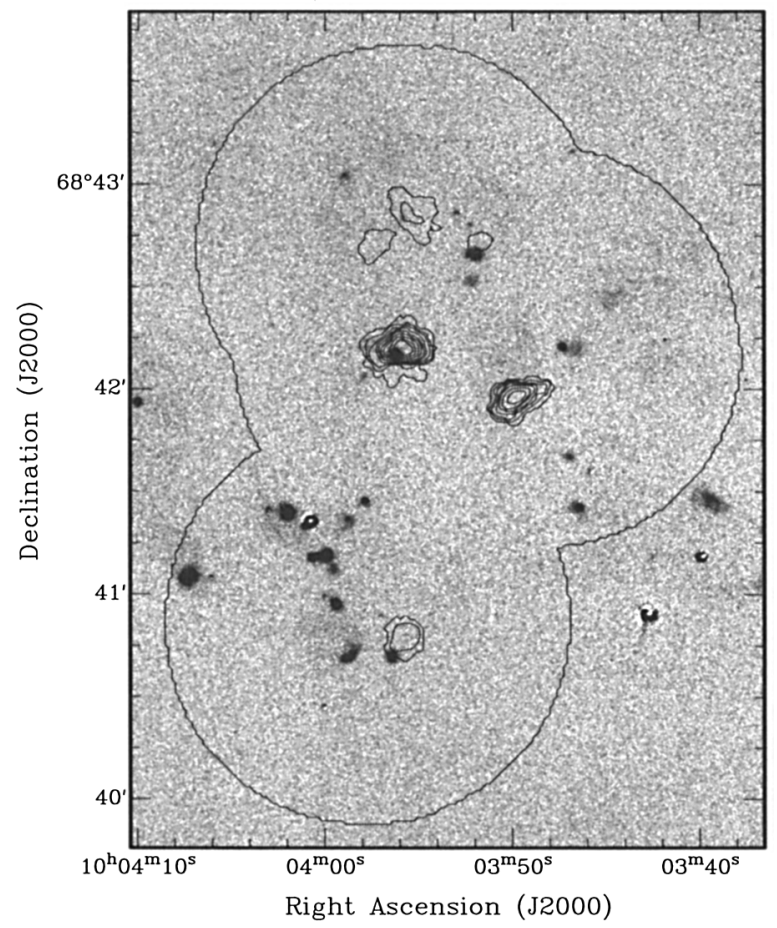

Figure 3. $\quad \mathrm{H} \alpha$ image of the region mapped with the OVRO millimeter interferometer. The contours represent the $\mathrm{CO}(1-0)$ emission at $5^{\prime \prime}$ $(75 \mathrm{pc})$ resolution. Note that the interferometer observations are only sensitive to clumped structures which is why the distribution looks different from Fig. 2.

formation is only seen where the HI surface brightness density reaches values larger than $\sim 1 \times 10^{21} \mathrm{~cm}^{-2}$ - this value is consistent with what is usually found in dwarf galaxies (see the discussion in Walter \& Brinks 1999). Star formation in the tidal arm seems to be suppressed where the HI kinematics show multiple velocity components. This may imply that the actual volume density of the atomic gas in these regions is not high enough to form molecular gas.

It is striking that the HII regions are predominantly situated at the southern edge of the tidal HI feature. This morphology may be indicative of bow-shock triggering in that region. We note that indications for bow-shocks in the southeast have been recently reported in two other dwarf galaxies in the same group (Holmberg I: Ott et al. 2001, Holmberg II: Bureau \& Carignan 2002).

It is difficult to estimate when star formation started in the tidal arm. From the fact that we see $\mathrm{H} \alpha$ emission today it is clear that star formation is occurring in the tidal arm well after the creation of the structure itself some $\sim 3 \times 10^{8}$ yrs ago ('in situ' formation). If we assume a constant star formation rate over the last $3 \times 10^{8}$ yr we estimate that a total of $\sim 7 \times 10^{5} \mathrm{M}_{\odot}$ have been transformed from gas into stars. Some of the fainter stars in the region may have therefore 
been created within the tidal feature itself. New HST ACS observations will enable us to test this hypothesis.

The presence of a huge reservoir of molecular as well as atomic gas suggests that star formation has just started in the tidal tail and that all the ingredients for further, more massive, star formation are present in this region. If some 10 percent of the gas will be transformed into stars, the total luminosity of the tidal dwarf will eventually be of order $5 \times 10^{7} \mathrm{M}_{\mathrm{B} \odot}$ (adopting a stellar mass-to-light ration of $\left.1 \mathrm{M}_{\odot} / \mathrm{L}_{\odot}\right)$, similar to other dwarf galaxies in the M 81 group. We conclude that wide-spread low-level star formation may be a common phenomenon in tidal HI tails, however it will be difficult to detect in typical, further-away interacting systems.

\section{References}

Brouillet, N., Baudry, A., Combes, F., Kaufman, M., \& Bash, F. 1991,A\&A, 242,35

Bureau, M. \& Carignan, C., 2002, AJ, 123,1316

Devereux, N., Duric, N., \& Scowen, P. A. 1997, AJ, 113, 236

Duc, P.-A., Brinks, E., Springel, V., Pichardo, B., Weilbacher, P., \& Mirabel, I. F. 2000, AJ, 120, 1238

Freedman, W.L., Hughes, S.M., Madore, B.F., Mould, J.R., Lee, M.G., Stetson, P., Kennicutt, R.C., Turner, A., Ferrarese, L., Ford, H., Graham, J.A., Hill, R., Hoessel, J.G., Huchra, J., Illingworth, G.D. 1994, ApJ, 427, 628

Heithausen, A. \& Walter, F. 2000, A\&A, 361, 500

Hunsberger, S. D., Charlton, J. C., \& Zaritsky, D. 1996, ApJ, 462, 50

Hunter, D. A., Hunsberger, S. D., \& Roye, E. W. 2000, ApJ, 542, 137

Karachentsev, I. D., Karachentseva, V. E., \& Boerngen, F. 1985, MNRAS, 217, 731

Kennicutt, R. C. 1983, ApJ, 272, 54

Leitherer, C. et al. 1999, ApJS, 123, 3

Miller, B. W. \& Hodge, P. 1994, ApJ, 427, 656

Mirabel, I. F., Dottori, H., \& Lutz, D. 1992, A\&A, 256, L19

Okazaki, T. \& Taniguchi, Y. 2000, ApJ, 543, 149

Rand, R. J. 1993, ApJ, 410, 68

Sakai, S., \& Madore, B., 2001, ApJ, 555, 280

Sharina, M. E. 1991, Soviet Astronomy Letters, 17, 383

Thomasson, M. \& Donner, K. J. 1993, A\&A, 272, 153

Yun, M. S., Ho, P. T. P., Brouillet, N., \& Lo, K. Y. 1993, in Evolution of Galaxies and their Environment, 253

Yun, M., Ho, P. \& Lo, K.Y., 1994, Nature, 372, 530

Walter, F. \& Brinks, E. 1999, AJ, 118, 273

Walter, F. \& Heithausen, A. 1999, ApJ, 519, L69

Walter, F., Weiss, A., Martin, C., Scoville, N., 2002, AJ, 123, 255

Yun, M. S., Ho, P. T. P., \& Lo, K. Y. 1994, Nature, 372, 530 